\title{
Expression and clinical significance of circular RNA hsa_circ_0079787 in the peripheral blood of patients with axial spondyloarthritis
}

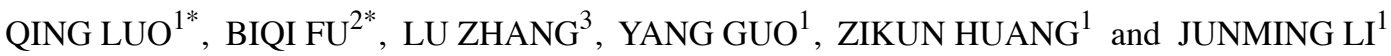 \\ Departments of ${ }^{1}$ Clinical Laboratory and ${ }^{2}$ Rheumatology, The First Affiliated Hospital of Nanchang \\ University; ${ }^{3}$ Medical College, Nanchang University, Nanchang, Jiangxi 330006, P.R. China
}

Received February 23, 2020; Accepted August 18, 2020

DOI: $10.3892 / \mathrm{mmr} .2020 .11520$

\begin{abstract}
Axial spondyloarthritis (AxSpA) is a chronic rheumatic disease involving the axial skeleton. Recent evidence suggested that certain circular RNAs (circRNAs) have a crucial role in rheumatic diseases. However, the functions of circRNAs in AxSpA have remained largely elusive. The present study identified the utility of the circRNA Homo sapiens (hsa)_circ_0079787 as a potential biomarker for AxSpA. A total of 5 circRNAs (hsa_circ_0002715, hsa_circ_0001947, hsa_circ_0079787, hsa_circ_0000367 and hsa_circ_0035197) were determined in the peripheral blood of 46 patients with $\mathrm{AxSpA}, 46$ patients with systemic lupus erythematosus (SLE) and 25 healthy controls (HC) by reverse transcription-quantitative PCR analysis. The detailed clinical history of each patient was recorded and the correlations between these circRNAs and clinical characteristics were analyzed. Furthermore, receiver operating characteristic (ROC) curves were constructed to evaluate the diagnostic value of hsa_circ_0079787 and other factors for AxSpA. Of the 5 selected circRNAs, the expression of hsa_circ_0079787 was indicated to be significantly reduced in the peripheral blood of patients with AxSpA as compared with the levels in HCs and patients with SLE. The peripheral blood levels of hsa_circ_0079787 in patients with AxSpA were negatively correlated with the Bath Ankylosing Spondylitis Disease Activity Index and positively correlated with the platelet count (PLT) and the lymphocyte-to-monocyte ratio. In addition, the expression of peripheral blood hsa_circ_0079787
\end{abstract}

Correspondence to: Dr Junming Li or Dr Zikun Huang, Department of Clinical Laboratory, The First Affiliated Hospital of Nanchang University, 17 Yongwai Zheng Street, Nanchang, Jiangxi 330006, P.R. China

E-mail: lisir361@163.com

E-mail: 491353062@qq.com

*Contributed equally

Key words: axial spondyloarthritis, circular RNAs, biomarker mmr.2020.11520 male patients with AxSpA was negatively correlated with the mean platelet volume (MPV) and positively correlated with the plateletcrit (PCT). ROC curve analysis suggested that hsa_circ_0079787 and the combination of hsa_circ_0079787-PLT-MPV-PCT had a significant diagnostic value for AxSpA.hsa_circ_0079787 and the combination of hsa_circ_0079787-PLT-MPV-PCT was also able to differentiate between patients with AxSpA and those with SLE. In conclusion, peripheral-blood hsa_circ_0079787 and the combination of hsa_circ_0079787-PLT-MPV-PCT may provide improved diagnostic accuracy for AxSpA. In addition, the levels of hsa_circ_0079787 in the peripheral blood were correlated with disease activity and severity of AxSpA.

\section{Introduction}

Axial spondyloarthritis (AxSpA) is a type of chronic inflammatory rheumatic disease involving the axial skeleton with a prevalence of $0.1-1.4 \%$ (1). Patients with AxSpA suffer from axial symptoms, resulting in fatigue, pain and limited mobility of the spine, which decreases their quality of life (2). AxSpA mainly affects individuals prior to the age of 45 years and the rate of early diagnosis is low. While the human leukocyte antigen (HLA)-B27 is the best genetic biomarker for diagnosing AxSPA and C-reactive protein (CRP) is the best circulating biomarker for assessing disease activity and predicting therapeutic responsiveness and structural progress discovered so far, their diagnostic value and their value for monitoring disease activity for AxSpA is low (3). Therefore, it is urgently required to identify novel effective and reliable markers for diagnosis and for monitoring disease activity to improve the survival of patients with AxSpA.

Circular RNA (circRNA) is a unique type of RNA, which is composed of exons, introns or products of reverse splicing of the two (4). As circRNAs do not have 5' or 3' ends, they are able to withstand RNase digestion and are more stable than most linear RNAs (5). In addition to their characteristic of comparatively higher stability, circRNAs frequently exhibit tissue/developmental stage-specific expression (6,7), and they are thus more suitable as biomarkers than linear RNAs $(6,8)$. Previous studies have confirmed that circRNAs may control gene transcription by isolating target microRNAs (miRNAs) 
and regulating RNA binding proteins, thus functioning as 'miRNA sponges' (9). Increasing evidence has indicated that certain circRNAs may be associated with the risk of nervous diseases, atherosclerotic vascular diseases, prion diseases, cancer and autoimmune diseases (10-13), which supports the hypothesis that circRNAs have the potential to become novel diagnostic and prognostic biomarkers and novel therapeutic targets for diseases $(14,15)$. However, the current knowledge of circRNAs in peripheral blood in patients with AxSpA is limited.

A previous study by our group identified certain differentially expressed circRNAs in the peripheral blood of patients with systemic lupus erythematosus (SLE) by circRNA microarray screening (16), suggesting that circRNAs may have a role in rheumatic diseases. Considering that SLE and AxSpA are common rheumatic diseases, although they have different pathogeneses, they have similar pathological and immunological abnormalities, and certain specific aberrant circRNAs (hsa_circ_0002715, hsa_circ_0001947, hsa_circ_0079787, hsa_circ_0000367 and hsa_circ_0035197) were selected to evaluate whether circRNAs may be used as novel diagnostic biomarkers for AxSpA. The present study aimed to determine whether these aberrant circRNAs in the peripheral blood may be used as novel diagnostic biomarkers for distinguishing patients with new-onset AxSpA from patients with SLE and healthy controls (HC).

\section{Materials and methods}

Participants. A total of 46 patients with new-onset AxSpA receiving clinical care at the Department of Rheumatology of the First Affiliated Hospital of Nanchang University (Nanchang, China) between September 2018 and March 2019 were enrolled in the present study. All patients with AxSpA fulfilled the Assessment of Spondyloarthritis international Society classification criteria for AxSpA (17). Those patients with AxSpA accompanied by other autoimmune, inflammatory or hormonal diseases, cancer or mental disorders were excluded. Prior to specimen collection, the patients with new-onset AxSpA did not receive any therapy with corticosteroids, disease-modifying anti-rheumatic drugs (DMARDs) or tumor necrosis factor inhibitors (TNFi). The peripheral blood of 25 age- and sex-matched HC who were free from autoimmune or inflammatory diseases and who were unrelated to the patients were recruited from the Physical Examination Center of the First Affiliated Hospital of Nanchang University (Nanchang, China) between September 2018 and March 2019. In addition, 46 patients with SLE who fulfilled the Systemic Lupus International Collaborating Clinics criteria (18) who presented at the First Affiliated Hospital of Nanchang University (Nanchang, China) at the same time were also enrolled. Among them, 27 patients had new-onset SLE that was diagnosed for the first time and they had no history of immunosuppressive drug or corticosteroids use prior to recruitment. Other subjects included were re-visiting SLE patients receiving treatment with $0.5-1 \mathrm{mg} / \mathrm{kg} / \mathrm{day}$ corticosteroids or $0.5-1.0 \mathrm{mg} / \mathrm{kg} / \mathrm{day}$ corticosteroids and immunosuppressive drugs, including $10-30 \mathrm{mg} / \mathrm{kg} /$ day mycophenolate mofetil, $0.75-1.0 \mathrm{~g} / \mathrm{m}^{2} / 3-4$ week cyclophosphamide, $3-5 \mathrm{mg} / \mathrm{kg} / \mathrm{day}$ cyclosporine, $0.5-1 \mathrm{mg} /$ day tacrolimus or $10-15 \mathrm{mg} /$ week methotrexate. All study protocols were approved by the Ethics Committee of the First Affiliated Hospital of Nanchang University (Nanchang, China; no. 019). All participants provided their written informed consent to participate in the study.

Preparation of peripheral blood samples and RNA isolation. Blood sample collection and total RNA isolation were performed as in a previous study by our group (19). Peripheral blood samples ( $2 \mathrm{ml}$ ) were collected in $\mathrm{K}_{2}$ EDTA-containing tubes and total RNA was extracted as soon as possible by using TRIzol reagent (Invitrogen; Thermo Fisher Scientific, Inc.) according to the manufacturer's protocol. The concentration and quality of the RNA were assessed by absorbance spectrometry to measure the ratios of absorbance at $260 \mathrm{~nm}$ (A260)/A280 and A260/A230 using a NanoDrop ND-1000 spectrophotometer (Thermo Fisher Scientific, Inc). Isolated total RNA was stored at $-80^{\circ} \mathrm{C}$ or immediately used for reverse transcription (RT).

RT-quantitative PCR (RT-qPCR) analysis. The methods outlined in a previous study by our group were followed (19). The primers used for $\mathrm{qPCR}$ are presented in Table I. $\beta$-actin was used as an internal control. The relative expression levels of each circRNA were determined using the equation $2^{-\Delta \Delta \mathrm{Cq}}(20)$.

Clinical assessments and laboratory indexes. AxSpA disease activity was measured using the Bath Ankylosing Spondylitis (AS) Functional Index (BASFI) and the Bath AS Disease Activity Index (BASDAI) $(21,22)$. HLA-B27 was detected by PCR. The erythrocyte sedimentation rate (ESR) was determined according to the manufacturer's protocol (Automatic measuring instrument for ESR XC-40B; Pu Li Sheng Corp.). $\mathrm{CRP}$ and $\mathrm{IgG}$ were measured by nephelometry. Blood routine parameters, including the white blood cell count (WBC), red blood cell count (RBC), hemoglobin (HGB), hematocrit (HCT), mean corpuscular volume (MCV), mean corpuscular hemoglobin ( $\mathrm{MCH}), \mathrm{MCH}$ concentration, red blood cell volume distribution width (RDW), platelet count (PLT), mean platelet volume (MPV), plateletcrit (PCT), platelet distribution width (PDW), lymphocyte count (L), lymphocyte percentage (L\%), monocyte count $(\mathrm{M})$, monocyte percentage (M\%), neutrophil count $(\mathrm{N})$, neutrophil percentage (N\%), eosinophil count, eosinophil percentage, basophil count, basophil percentage and platelet large-cell ratio were measured using the Sysmex XE-2100 analyzer (Sysmex Corp.).

Annotation of circRNA/miRNA interactions. The circRNA/miRNA interactions were predicted using Arraystar's home-made miRNA target prediction software (version 1; KangChen Bio-tech, Inc.) $(23,24)$ based on TargetScan (version 6) (25) and miRanda (version 3.3a) (26), and the differentially expressed circRNAs within all the comparisons were annotated in detail with the circRNA/miRNA interaction information.

Statistical analysis. Baseline characteristics were assessed using descriptive statistics. An unpaired Student's t-test and the Mann-Whitney U-test were employed to compare normally distributed parameters and those with a skewed 
Table I. Specific circRNA primers used for quantitative PCR analysis.

\begin{tabular}{ll}
\hline Name & \multicolumn{1}{c}{ Sequence $\left(5^{\prime} \rightarrow 3^{\prime}\right)$} \\
\hline hsa_circ_0002715 & F: GCAAACCTCCTCTCCATGCT \\
& R: GTGAAAAGGCTGTGCCTGTG \\
hsa_circ_0001947 & F: ACACTCTTGGATGGAAAACCCA \\
& R: CGTGTTCTGGACTCGGTTGG \\
hsa_circ_0079787 & F: AGAGGAAGTTTGATTGCACTCTG \\
& R: TTCACGGAGAGGTTGTGTCC \\
hsa_circ_0000367 & F: AGACTGGCGTGAAACCTTCC \\
& R: TGCACAGTGGATGGATCATGG \\
hsa_circ_0035197 & F: TAATCGGCAGTCTGAAGTGCT \\
& R: GGCATTTGCCACTCTTTGGA \\
\$-actin & F: CATGTACGTTGCTATCCAGGC \\
& R: CTCCTTAATGTCACGCACGAT
\end{tabular}

circRNA, circular RNA; F, forward; R, reverse; hsa, Homo sapiens.

distribution, respectively. Analysis of variance or the Kruskal-Wallis test were used to compare more than two groups, and Tukey's honestly-significant differences test or Dunn's test was used as the post-hoc test, respectively. The Spearman method was used for correlation analysis. In addition, receiver operating characteristic (ROC) curve analyses were performed to assess the diagnostic value of the circRNA Homo sapiens (hsa)_circ_0079787. A two-sided $\mathrm{P}<0.05$ was considered to indicate statistical significance. Statistical analysis and graphical presentation were performed with SPSS version 16.0 (SPSS, Inc.) and GraphPad Prism version 5.0 (GraphPad Software, Inc.).

\section{Results}

Characteristics of the study population. A total of 117 participants were enrolled in the present study from September 2018 to March 2019, including 46 patients with new-onset AxSpA, 46 patients with new-onset SLE and $25 \mathrm{HC}$. All patients with AxSpA had new-onset disease with no history of corticosteroid, DMARD or TNFi use prior to enrolment. Of the 46 patients with AxSpA, 32 patients with AxSpA were used for the initial analysis and 14 patients were used for validation. The demographic characteristics of the study population are provided in Table II. There was no significant difference in age between the new-onset AxSpA group, the HC group and the SLE group. Furthermore, there was no significant difference in gender between the new-onset AxSpA group and the HC group, while there was a difference between the new-onset AxSpA group and the SLE group. No association between hsa_circ_0079787 levels and age or gender was observed in the new-onset AxSpA group, HC group and SLE group (data not shown). In addition, the WBC, PLT, PCT, N, N\% and NLR were significantly higher in the AxSpA as compared with those in the HC group (all $\mathrm{P}<0.05$; Table II), while the MPV and L\% were significantly lower in the $\mathrm{AxSpA}$ as compared with those in the $\mathrm{HC}$ group (all $\mathrm{P}<0.05$; Table II). Furthermore, the WBC,
RBC, HGB, HCT, PLT, PCT, L and LMR were significantly higher in the AxSpA than those in the SLE group (all $\mathrm{P}<0.05$; Table II), while the ESR, MPV and M\% were significantly lower in the AxSpA as compared with those in the SLE group (all $\mathrm{P}<0.05$; Table II).

Peripheral blood hsa_circ_0079787 levels are decreased in patients with AxSpA. Hsa_circ_0002715, hsa_circ_0001947, hsa_circ_0079787, hsa_circ_0000367 and hsa_circ_0035197 were detected to compare their expression in the peripheral blood of 32 patients with AxSpA and $25 \mathrm{HC}$. As presented in Fig. 1, the levels of hsa_circ_0079787 were significantly decreased in the peripheral blood of patients with $\mathrm{AxSpA}$ as compared with those in the HC group $(\mathrm{P}=0.0395)$. However, the peripheral blood levels of the other circRNAs were not significantly different between the patients with AxSpA and the $\mathrm{HC}$ (all $\mathrm{P}>0.0500)$.

Validation of peripheral blood levels of hsa_circ_0079787 in the second cohort. Next, the expression of hsa_circ_0079787 in the peripheral blood was verified in an independent cohort of 14 patients with $\mathrm{AxSpA}$ and the levels were significantly decreased as compared with those in the $25 \mathrm{HC}(\mathrm{P}=0.0006$; Fig. 2A). Analysis of all of the 46 patients with AxSpA and $\mathrm{HC}$ also indicated that the expression of hsa_circ_0079787 in the peripheral blood of the patients with AxSpA was significantly decreased as compared with that in the $25 \mathrm{HC}$ $(\mathrm{P}=0.0030$; Fig. 2B).

Correlation of peripheralblood hsa_circ_0079787 levels with clinical characteristics of patients with AxSpA. To investigate whether the peripheral blood levels of hsa_circ_0079787 may serve as a biomarker for disease activity and severity in patients with $\mathrm{AxSpA}$, the correlations between the peripheral blood levels of hsa_circ_0079787 in patients with AxSpA and their laboratory parameters, including BASFI, BASDAI, HLA-B27, ESR, CRP, IgG, WBC, RBC, HGB, HCT, PLT, MPV, PCT, PDW, L, L\%, M, M\%, N, N\%, NLR, PLR and LMR, were investigated. As presented in Fig. 3, the peripheral blood levels of hsa_circ_0079787 in patients with AxSpA were negatively correlated with the BASDAI [Spearman correlation coefficient $\left.\left(r_{s}\right)=-0.4737, P=0.0126\right]$, while they were positively correlated with PLT $\left(\mathrm{r}_{\mathrm{s}}=0.3201, \mathrm{P}=0.0301\right)$ and LMR ( $\left.\mathrm{r}_{\mathrm{s}}=0.3088, \mathrm{P}=0.0368\right)$. In addition, the peripheral blood levels of hsa_circ_0079787 in male patients with AxSpA were negatively correlated with MPV $\left(\mathrm{r}_{\mathrm{s}}=-0.3486, \mathrm{P}=0.0433\right)$ and positively correlated with PCT $\left(r_{s}=0.4551, \mathrm{P}=0.0068\right)$.

Potential diagnostic value of peripheral blood hsa_ circ_0079787 in patients with AxSpA. Various studies have indicated that circRNAs may serve as promising biomarkers for disease detection. Therefore, an ROC curve was drawn to assess the diagnostic value of peripheral blood hsa circ_0079787 in discriminating patients with AxSpA from HC. As presented in Fig. 4, the area under the ROC curve (AUC) was up to 0.715 [95\% $\mathrm{CI}=0.583-0.847 ; \mathrm{P}=0.0030]$. The sensitivity, specificity and cut-off were $82.61 \%, 56.00 \%$ and 0.01287 , respectively. It was therefore indicated that the peripheral blood levels of hsa_circ_0079787 may be used as a diagnostic biomarker for AxSpA. 
Table II. Clinical details of the patients with AxSpA, HC and SLE patients.

\begin{tabular}{|c|c|c|c|}
\hline Clinical characteristic & $\operatorname{AxSpA}(n=46)$ & $\mathrm{HC}(\mathrm{n}=25)$ & SLE $(n=46)$ \\
\hline Sex (male/female) & $34 / 12$ & $15 / 10$ & $2 / 44$ \\
\hline Age (years) & $32.59 \pm 12.39$ & $37.64 \pm 9.74$ & $34.41 \pm 16.01$ \\
\hline BASFI & $2.20 \pm 2.29$ & & \\
\hline BASDAI & $3.50 \pm 1.82$ & & \\
\hline $\begin{array}{l}\text { HLA-B27 positive (out } \\
\text { of } 28 \text { patients tested) }\end{array}$ & 21 & & \\
\hline $\operatorname{ESR}(\mathrm{mm} / \mathrm{h})$ & $24.00 \pm 26.23$ & & $49.76 \pm 33.05^{\mathrm{a}}$ \\
\hline CRP (mg/l) & $13.98 \pm 19.25$ & & $10.04 \pm 25.41$ \\
\hline WBC $\left(10^{9} / 1\right)$ & $7.57 \pm 1.97^{b}$ & $5.82 \pm 1.16$ & $6.45 \pm 3.65^{\mathrm{a}}$ \\
\hline $\mathrm{RBC}\left(10^{12} / 1\right)$ & $4.86 \pm 0.68$ & $4.76 \pm 0.43$ & $3.69 \pm 0.89^{\mathrm{a}}$ \\
\hline HGB (g/l) & $143.37 \pm 20.01$ & $146.64 \pm 11.31$ & $106.24 \pm 27.30^{\mathrm{a}}$ \\
\hline $\operatorname{HCT}(1 / 1)$ & $0.44 \pm 0.06$ & $0.43 \pm 0.03$ & $0.32 \pm 0.08^{\mathrm{a}}$ \\
\hline $\operatorname{PLT}\left(10^{9} / 1\right)$ & $317.46 \pm 92.79^{\mathrm{b}}$ & $249.92 \pm 49.39$ & $201.98 \pm 88.85^{\mathrm{a}}$ \\
\hline MPV (fl) & $9.73 \pm 1.01^{\mathrm{b}}$ & $10.81 \pm 0.96$ & $10.68 \pm 1.15^{\mathrm{a}}$ \\
\hline $\operatorname{PCT}(\%)$ & $0.31 \pm 0.07^{b}$ & $0.27 \pm 0.05$ & $0.22 \pm 0.08^{\mathrm{a}}$ \\
\hline PDW (fl) & $12.89 \pm 2.77$ & $13.07 \pm 2.20$ & $13.23 \pm 2.76$ \\
\hline $\mathrm{L}\left(10^{9} / 1\right)$ & $2.13 \pm 0.62$ & $1.95 \pm 0.43$ & $1.37 \pm 0.88^{\mathrm{a}}$ \\
\hline $\mathrm{L}(\%)$ & $27.75 \pm 7.92^{\mathrm{b}}$ & $33.69 \pm 4.99$ & $23.73 \pm 10.96$ \\
\hline $\mathrm{M}\left(10^{9} / 1\right)$ & $0.43 \pm 0.15^{\mathrm{b}}$ & $0.36 \pm 0.09$ & $0.47 \pm 0.38$ \\
\hline $\mathrm{M}(\%)$ & $5.67 \pm 1.53$ & $6.16 \pm 1.32$ & $7.43 \pm 3.00^{\mathrm{a}}$ \\
\hline $\mathrm{N}\left(10^{9} / 1\right)$ & $4.85 \pm 1.58^{\mathrm{b}}$ & $3.35 \pm 0.79$ & $4.55 \pm 3.08$ \\
\hline $\mathrm{N}(\%)$ & $63.39 \pm 7.71^{\mathrm{b}}$ & $57.37 \pm 5.72$ & $68.21 \pm 11.98^{c}$ \\
\hline PLR & $160.32 \pm 64.51^{\mathrm{d}}$ & $133.40 \pm 36.75$ & $190.71 \pm 121.77$ \\
\hline NLR & $2.44 \pm 0.95^{\mathrm{b}}$ & $1.77 \pm 0.47$ & $4.42 \pm 4.23^{\mathrm{c}}$ \\
\hline LMR & $5.48 \pm 2.32$ & $5.71 \pm 1.50$ & $3.58 \pm 1.78^{a}$ \\
\hline
\end{tabular}

${ }^{\mathrm{a}} \mathrm{P}<0.05$, SLE compared to AxSpA; ${ }^{\mathrm{b}}<0.05$, AxSpA compared to $\mathrm{HC},{ }^{\mathrm{C}} \mathrm{P}=0.05$, SLE compared to AxSpA; ${ }^{\mathrm{d}} \mathrm{P}=0.05$, AxSpA compared to HC. AxSpA, axial spondyloarthritis; HLA-B27, human leukocyte antigen; BASDAI, Bath Ankylosing Spondylitis Disease Activity Index; BASFI, Bath Ankylosing Spondylitis Functional Index; CRP, C-reactive protein; ESR, erythrocyte sedimentation rate; HC, healthy controls; HCT, hematocrit; HGB, hemoglobin; L, lymphocyte count; L\%, lymphocyte percentage; LMR, lymphocyte-to-monocyte ratio; M, monocyte count; M\%, monocyte percentage; MPV, mean platelet volume; N, neutrophil count; N\%, neutrophil percentage; NLR, neutrophil-to-lymphocyte ratio; PCT, plateletcrit; PDW, platelet distribution width; PLR, platelet-to-lymphocyte ratio; PLT, platelet count; RBC, red blood cell count; SLE, systemic lupus erythematosus; WBC, white blood cell count.

In addition, among the 46 patients with $\mathrm{AxSpA}$, 28 patients were tested for HLA-B27 and 21 patients were positive. All HC were tested for HLA-B27 and were negative. None of the patients with SLE were tested for HLA-B27. Using the cut-off value for hsa_circ_0079787 of $<0.01287$ and the result of the HLA-B27 test, the potential diagnostic value of the combination of hsa_circ_0079787-HLA-B27 to distinguish patients with $\mathrm{AxSpA}$ from $\mathrm{HC}$ was investigated. It was demonstrated that the combination model of hsa_circ_0079787-HLA-B27 was able to effectively discriminate patients with AxSpA from $\mathrm{HC}$ with a sensitivity of $96.43 \%(27 / 28)$, a specificity of $100.00 \%(25 / 25)$ and an accuracy of $98.11 \%$ (52/53) (Table III). The diagnostic value of the combination of hsa_circ_0079787-HLA-B27 for AxSpA vs. HC was superior to that of HLA-B27, which in turn had a sensitivity of $75.00 \%(21 / 28)$, a specificity of $100.00 \%(25 / 25)$ and an accuracy of $86.79 \%(46 / 53)$ (Table II).
Peripheral blood levels of hsa_circ_0079787 in patients with AxSpA and SLE. As presented in Fig. 5A, the peripheral blood levels of hsa_circ_0079787 were markedly decreased in patients with $\mathrm{AxSpA}$ as compared with those in patients with SLE.

Next, an ROC curve analysis of peripheral blood hsa circ_0079787 to distinguish patients with AxSpA from those with SLE was performed. The AUC for hsa_circ_0079787 to discriminate between patients with AxSpA and those with SLE was 0.694 , with a sensitivity of $71.79 \%$, a specificity of $67.39 \%$ and a cut-off value of $<0.01053$ (Fig. 5B). In addition, the peripheral blood levels of hsa_circ_0079787 were able to distinguish patients with AxSpA from HC and patients with SLE combined $(\mathrm{HC}+\mathrm{SLE})$. The AUC for hsa_circ_0079787 in patients with AxSpA vs. HC + SLE was 0.658 with a sensitivity of $82.61 \%$, a specificity of $49.3 \%$ and a cut-off value of $<0.01287$ (Fig. 5C).

As presented in Table I, the PLT, MPV and PCT in patients with AxSpA were different from those in the $\mathrm{HC}$ and SLE groups. Thus, the potential diagnostic value of the 

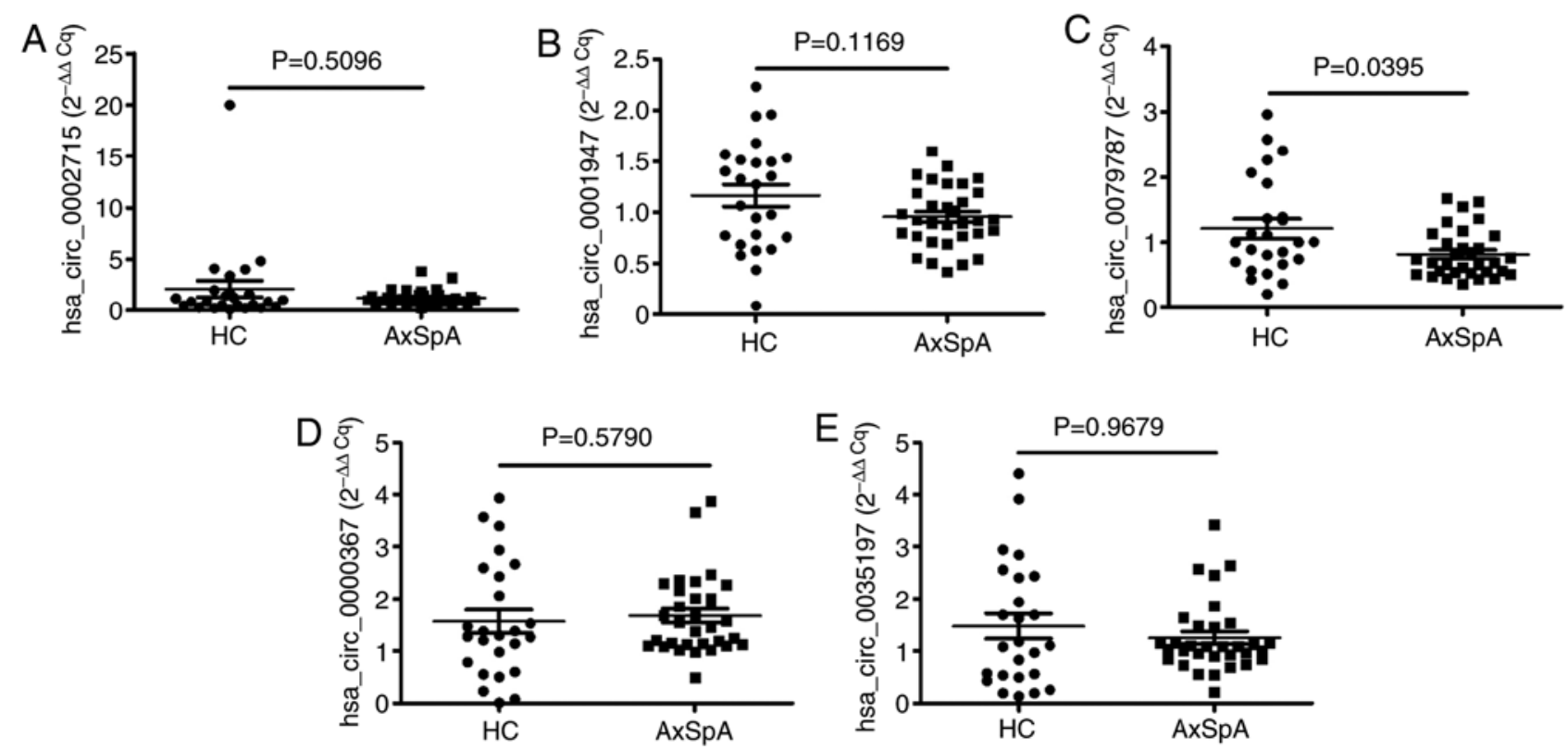

Figure 1. Screening of abnormally expressed circRNAs in peripheral blood from 32 patients with AxSpA and 25 HC. The expression of hsa_circ_0002715, hsa_ circ_0001947, hsa_circ_0079787, hsa_circ_0000367, hsa_circ_0035197 was determined in peripheral blood from 32 patients with AxSpA and 25 HC by reverse transcription-quantitative PCR analysis. (A) hsa_circ_0002715, (B) hsa_circ_0001947, (C) hsa_circ_0079787, (D) hsa_circ_0000367 and (E) hsa_circ_0035197 levels were compared between the groups. Each dot represents an individual patient. Differences were evaluated with the Mann-Whitney U-test. AxSpA, axial spondyloarthritis; Cq, quantification cycle; HC, healthy controls; circRNA/circ, circular RNA; hsa, Homo sapiens.
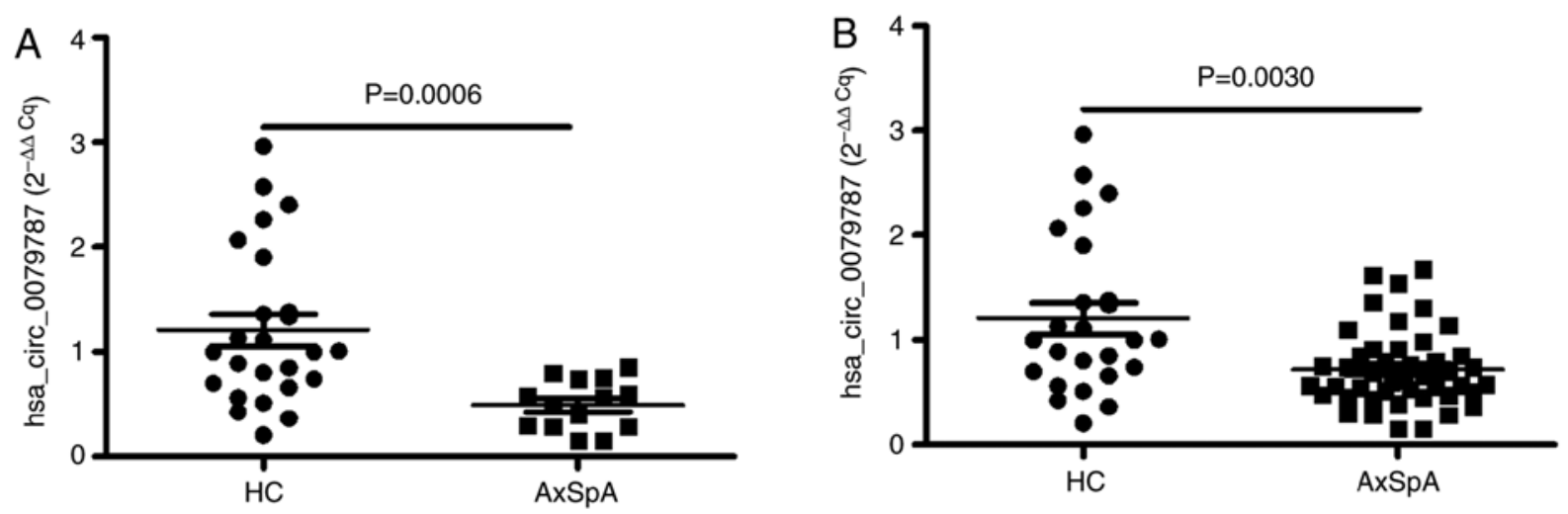

Figure 2. Validation of the expression of hsa_circ_0079787 in peripheral blood from patients with AxSpA. The expression of hsa_circ_0079787 was determined in the peripheral blood from (A) an independent cohort of 14 cases and (B) all 46 patients with AxSpA, as well as the $25 \mathrm{HC}$, by reverse transcription-quantitative PCR analysis (Mann-Whitney U-test). Each dot represents an individual patient. Differences were evaluated with the Mann-Whitney U-test. AxSpA, axial spondyloarthritis; HC, healthy controls; circRNA/circ, circular RNA; hsa, Homo sapiens.

combination of hsa_circ_0079787-PLT-MPV-PCT for AxSpA and SLE was investigated. The AUC for the combination of hsa_circ_0079787-PLT-MPV-PCT to distinguish patients with AxSpA from HC was 0.835, with a sensitivity of $73.91 \%$ and a specificity of $80.00 \%$ (Fig. 5D). Furthermore, the AUC for the combination of hsa_circ_0079787-PLT-MPV-PCT to distinguish patients with AxSpA from those with SLE was 0.836 , with a sensitivity of $73.91 \%$ and a specificity of $82.50 \%$ (Fig. 5E). The AUC for the combination of hsa circ_0079787-PLT-MPV-PCT to distinguish patients with AxSpA patients from SLE + HC subjects was 0.838 , with a sensitivity of $73.91 \%$ and a specificity of $81.54 \%$ (Fig. 5F).

Target miRNA prediction for hsa_circ_0079787. To confirm the function of hsa_circ_0079787, potential miRNA targets (hsa-miR-580-3p, hsa-miR-125-5p, hsa-miR-5191, hsa-miR-6830-5p and hsa-miR-4694-3p) of the circRNAs were predicted by aligning with the miRNA response elements using TargetScan and miRanda software. Details of the relationships between hsa_circ_0079787 and the above target miRNAs are presented in Fig. 6 .

\section{Discussion}

CircRNAs are a special class of endogenous RNAs. Over the past few years, an increasing number of studies have significantly expanded the current knowledge of circRNAs, including their function, expression and utility as diagnostic or predictive biomarkers in rheumatic diseases, including SLE and rheumatic arthritis (RA) (13,19,27-29). A previous study by our 
A

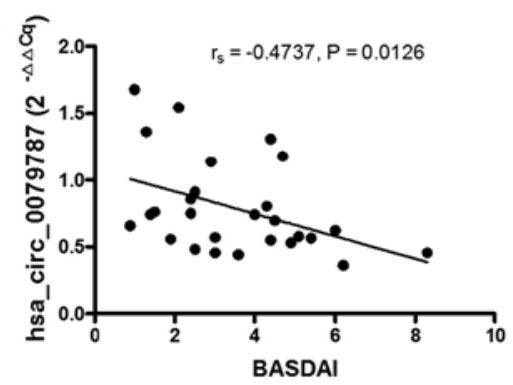

B

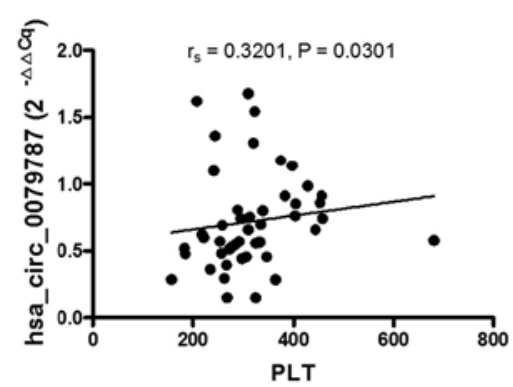

C

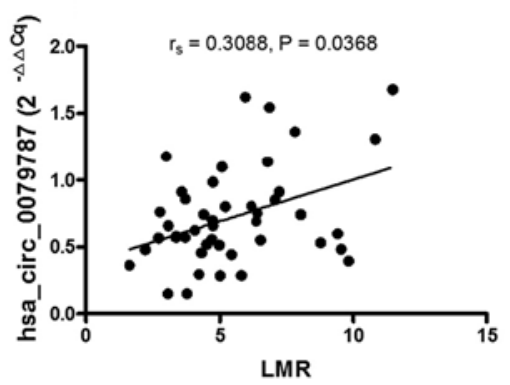

D

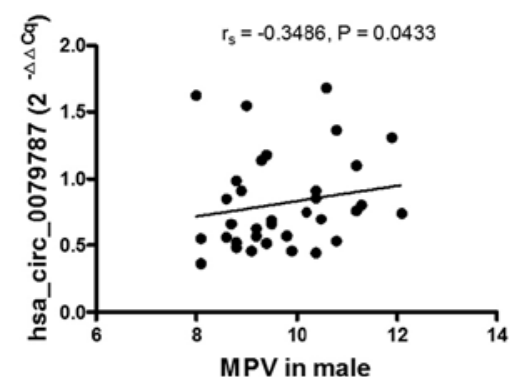

$\mathrm{E}$

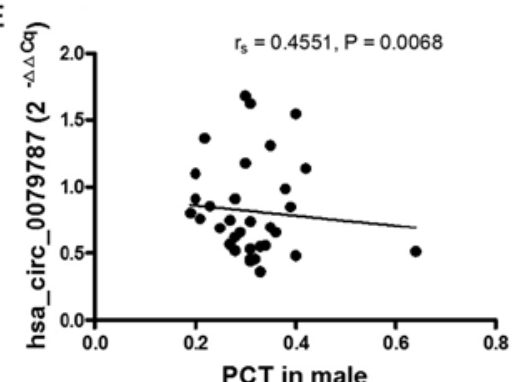

Figure 3. Correlation of peripheral blood hsa_circ_0079787 levels in patients with AxSpA with clinical characteristics. The BASDAI, LMR, MPV, PCT and PLT were measured. Correlations of peripheral blood hsa_circ_007978 levels in patients with AxSpA with (A) BASDAI, (B) PLT, (C) LMR, (D) MPV in male and (E) PCT in male were analyzed with Spearman's non-parametric test. Each dot represents an individual patient. AxSpA, axial spondyloarthritis; circRNA/circ, circular RNA; hsa, Homo sapiens; BASDAI, Bath Ankylosing Spondylitis Disease Activity Index; LMR, lymphocyte-to-monocyte ratio; MPV, mean platelet volume; PCT, plateletcrit; PLT, platelet count; $r_{s}$, Spearman correlation coefficient.

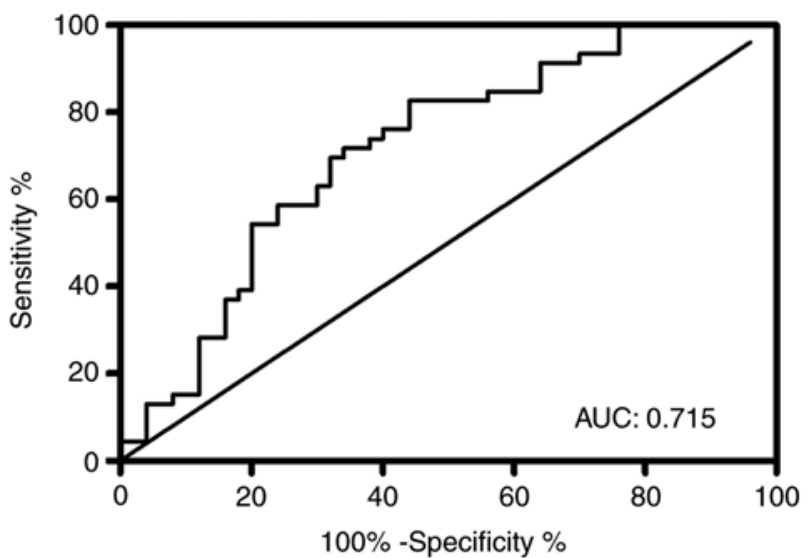

Figure 4. ROC curve analysis of peripheral blood hsa_circ_0079787 in patients with AxSpA. The ROC curve was constructed to evaluate the ability of hsa_circ_0079787 to distinguish patients with AxSpA from healthy controls. ROC, receiver operating characteristic; AUC, area under the ROC curve; AxSpA, axial spondyloarthritis; circRNA/circ, circular RNA; hsa, Homo sapiens.

group demonstrated that the combination of hsa_circ_0002715 and hsa_circ_0035197 in peripheral blood may be a potential biomarker for new-onset RA and to be associated with disease activity (29). However, the expression of circRNAs in patients with AxSpA had remained largely elusive. In the present study, RT-qPCR analysis was utilized to detect the expression of circRNAs (hsa_circ_0002715, hsa_circ_0001947, hsa_circ_0079787, hsa_circ_0000367 and hsa_circ_0035197) in the peripheral blood of patients with AxSpA and HC, and only hsa_circ_0079787 was significantly decreased in patients with AxSpA, suggesting that hsa_circ_0079787 in peripheral blood was associated with AxSpA.
Various studies have indicated that circRNAs may serve as indicators of disease activity and severity. Miao et al (30) demonstrated that downregulation of circ protein tyrosine phosphatase non-receptor type 22 (PTPN22), which is the PTPN22, in the peripheral blood mononuclear cells of patients with SLE was negatively correlated with the SLE disease activity index (SLEDAI) score and circPTPN22 was proved to be an activity indicator in SLE. Ouyang et al (31) indicated that elevated plasma circRNA_002453 was significantly and positively correlated with 24-h proteinuria and the renal SLEDAI score in patients with lupus nephritis, which suggested that upregulated plasma circRNA_002453 levels were associated with the severity of renal involvement. In the present study, the clinical manifestations (BASDAI, BASFI) and laboratory indicators (HLA-B27, inflammation markers, immune cells) of patients with AxSpA were different from the controls, and these parameters may indicate the activity and severity of AxSpA. Spearman's rank correlation analysis revealed that hsa_circ_0079787 in peripheral blood of patients with AxSpA was negatively correlated with the BASDAI, which mirrors the activity of AxSpA (21). Furthermore, the expression of peripheral blood hsa_circ_0079787 was positively correlated with LMR in patients with AxSpA. In rheumatic diseases, LMR is known to be associated with disease severity (32). These results indicated that hsa_circ_0079787 in peripheral blood may be used as an indicator of disease activity and severity of AxSpA.

In addition, the correlation between PLT-associated parameters (PLT, PCT and MPV) and the expression of peripheral blood hsa_circ_0079787 in AxSpA was investigated and it was indicated that the expression of peripheral blood hsa_circ_0079787 was correlated with PLT in patients with AxSpA, as well as with MPV and PCT in male patients 
Table III. Diagnostic efficiency of the combination of hsa_circ_0079787-HLA-B27 for AxSpA.

A, hsa_circ_0079787 $<0.01287$ or HLA-B27 positive

\begin{tabular}{|c|c|c|c|c|c|}
\hline Categories & Positive & Negative & Sensitivity & Specificity & Accuracy \\
\hline HLA-B27 (28) & 27 & 1 & $96.43 \%(27 / 28)$ & $100.00 \%(25 / 25)$ & $98.11 \%(52 / 53)$ \\
\hline $\mathrm{HC}(25)$ & 0 & 25 & & & \\
\hline
\end{tabular}

B, HLA-B27 positive

\begin{tabular}{|c|c|c|c|c|c|}
\hline Categories & Positive & Negative & Sensitivity & Specificity & Accuracy \\
\hline HLA-B27 (28) & 21 & 7 & $75.00 \%(21 / 28)$ & $100.00 \%(25 / 25)$ & $86.79 \%(46 / 53)$ \\
\hline $\mathrm{HC}(25)$ & 0 & 25 & & & \\
\hline
\end{tabular}

AxSpA, axial spondyloarthritis; HLA-B27, human leukocyte antigen; HC, healthy control.
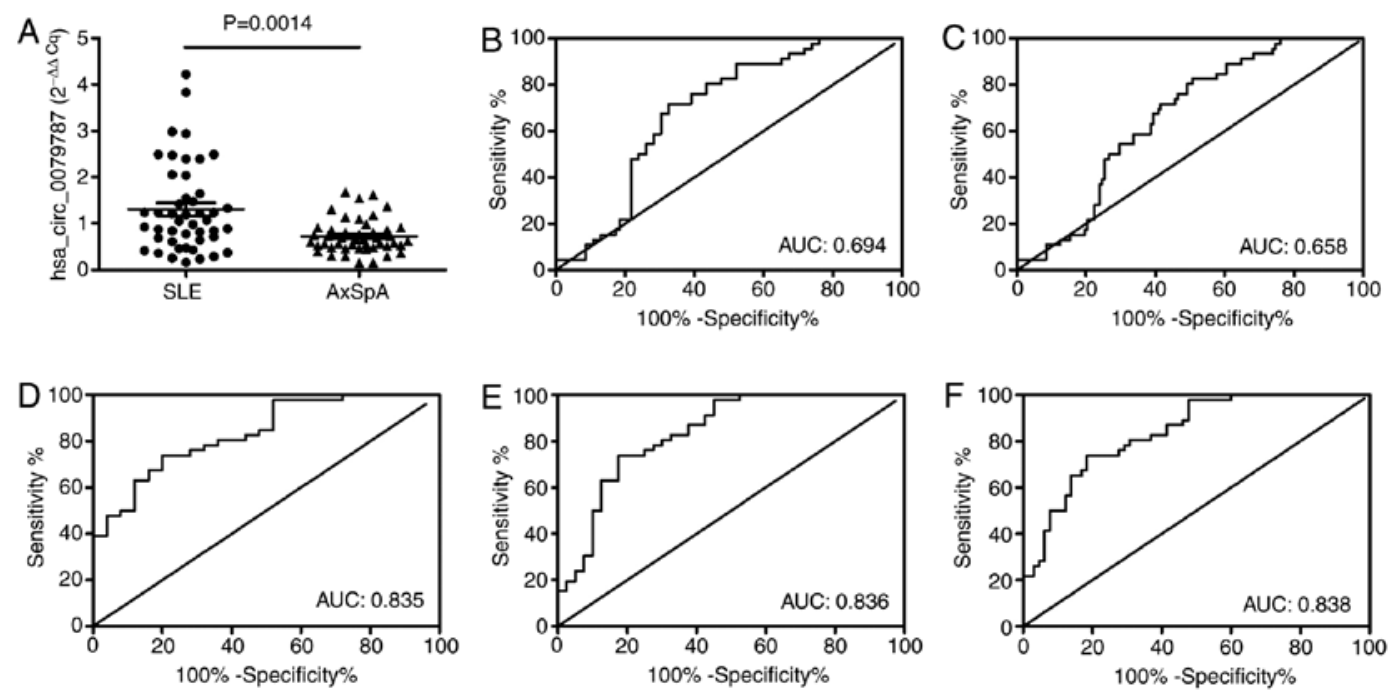

Figure 5. ROC curve analysis for peripheral blood hsa_circ_0079787 and the combination of hsa_circ_0079787-PLT-MPV-PCT as a risk score to distinguish between AxSpA and SLE or HC or SLE + HC. The expression of hsa_circ_0079787 was determined in the peripheral blood of 46 patients with AxSpA and 46 patients with SLE by reverse transcription-quantitative PCR analysis. ROC curves were constructed to evaluate the diagnostic value of hsa_circ_0079787 for AxSpA. (A) Comparison of hsa_circ_0079787 levels between patients with AxSpA and SLE (Mann-Whitney U-test) and (B-F) ROC curve analysis for (B) hsa_circ_0079787 in AxSpA vs. SLE, (C) hsa_circ_0079787 in AxSpA vs. SLE + HC, (D) the combination of hsa_circ_0079787-PLT-MPV-PCT in AxSpA vs. HC, (E) the combination of hsa_circ_0079787-PLT-MPV-PCT in AxSpA vs. SLE and (F) the combination of hsa_circ_0079787-PLT-MPV-PCT in AxSpA vs. SLE + HC. Each dot represents an individual patient. ROC, receiver operating characteristic; AxSpA, axial spondyloarthritis; HC, healthy controls; SLE, systemic lupus erythematosus; circRNA/circ, circular RNA; MPV, mean platelet volume; PCT, plateletcrit; PLT, platelet count; hsa, Homo sapiens.

with AxSpA. Although the activation of platelets was reported to be associated with disease progression and the PLT is increased during the active stage of AS (33), the MPV was negatively correlated with the PLT in AS (34), and evidence from a study on other rheumatic diseases indicated that the PLT and PCT were associated with disease severity (35). AS is a prototype of $\mathrm{AxSpA}$ and is characterized as damage seen on radiography. However, the association between PLT-related parameters and disease activity in patients with AxSpA has remained largely elusive; of note, the present study suggested that PLT-associated parameters (PLT, PCT and MPV) were not associated with the activity of AxSpA (BASDAI and BASFI; data no shown). Thus, the peripheral blood levels of hsa_circ_0079787 in AxSpA were associated with PLT-related parameters (PLT, PCT and MPV) that may have different roles at different stages of AxSpA.

Furthermore, there were no correlations between hsa_circ_0079787 and CRP or ESR. Although CRP and ESR are routine inflammatory biomarkers, CRP is not elevated in a large proportion of patients with active AxSpA (3) and its correlation with the disease activity, including the BASFI, is inconclusive. In addition, ESR is a nonspecific measure of inflammation that may be influenced by a variety of other conditions and its value as a marker of disease activity appears to be poorer than that of CRP (3).

In the present study, the ROC curve analysis indicated that hsa_circ_0079787 in the peripheral blood was able to distinguish patients with AxSpA from HC with an AUC of 0.715, high 
hsa-miR-580-3p

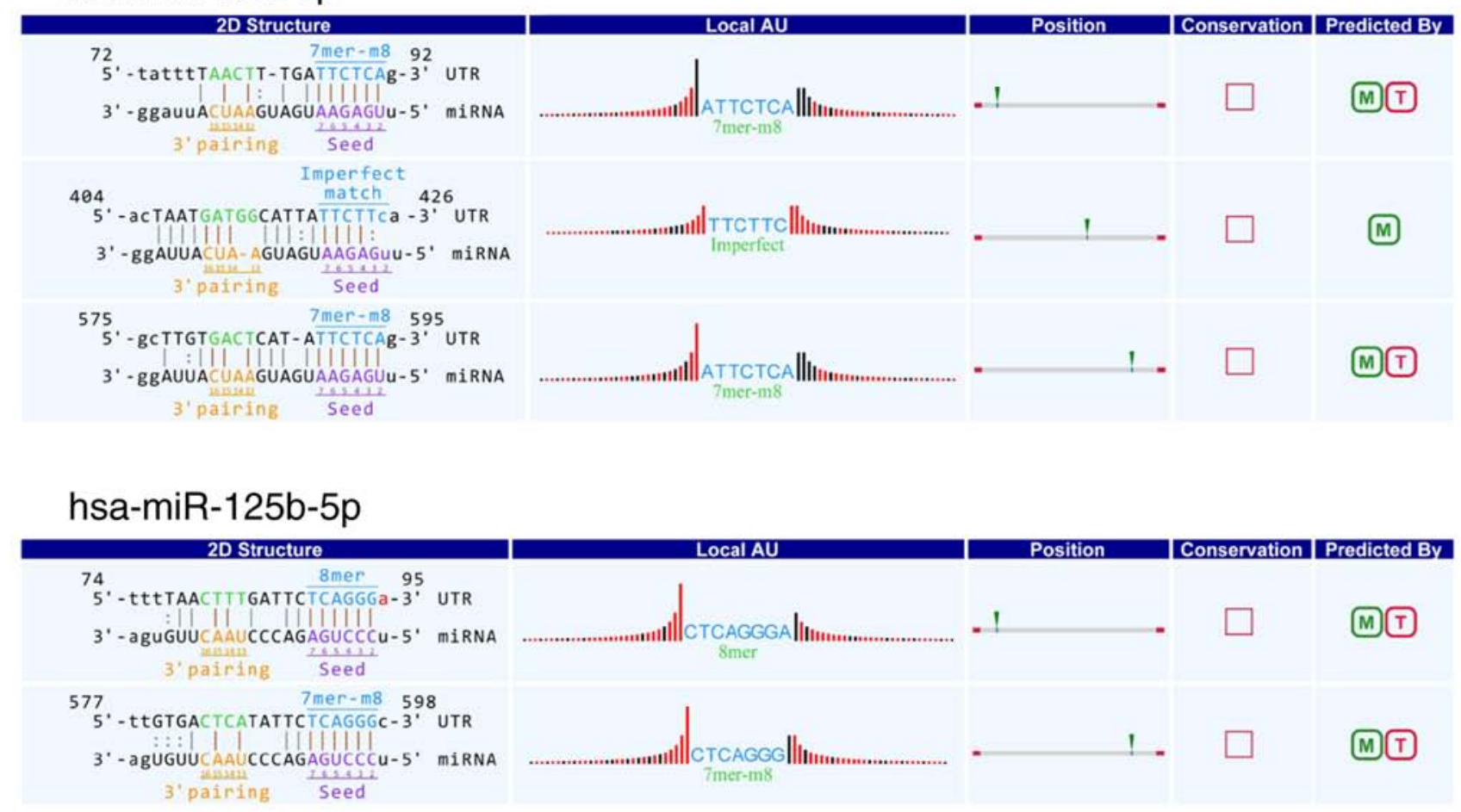

hsa-miR-5191

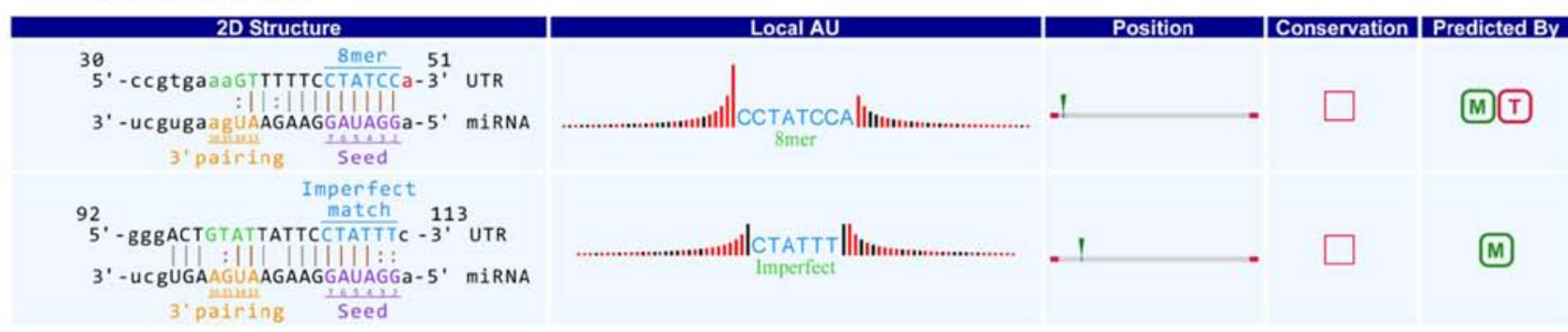

\section{hsa-miR-6830-5p}

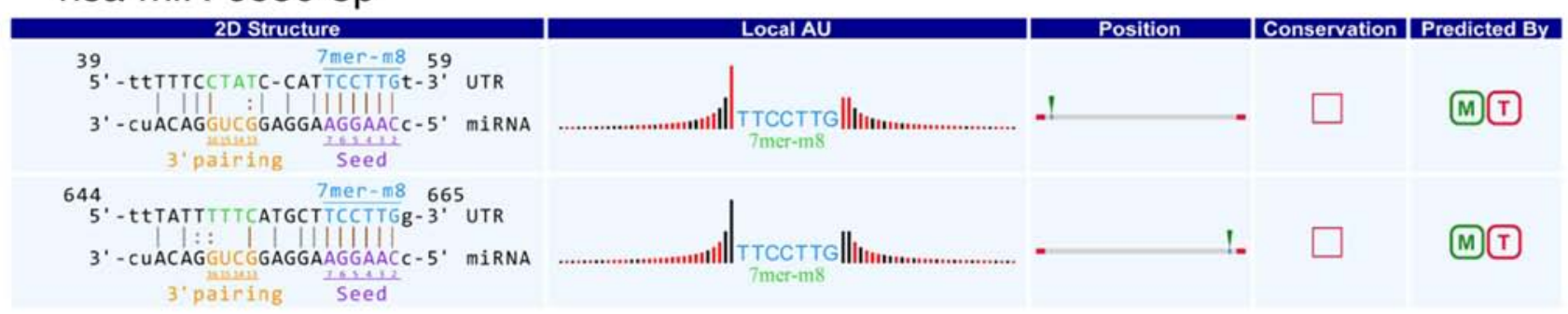

\section{hsa-miR-4694-3p}

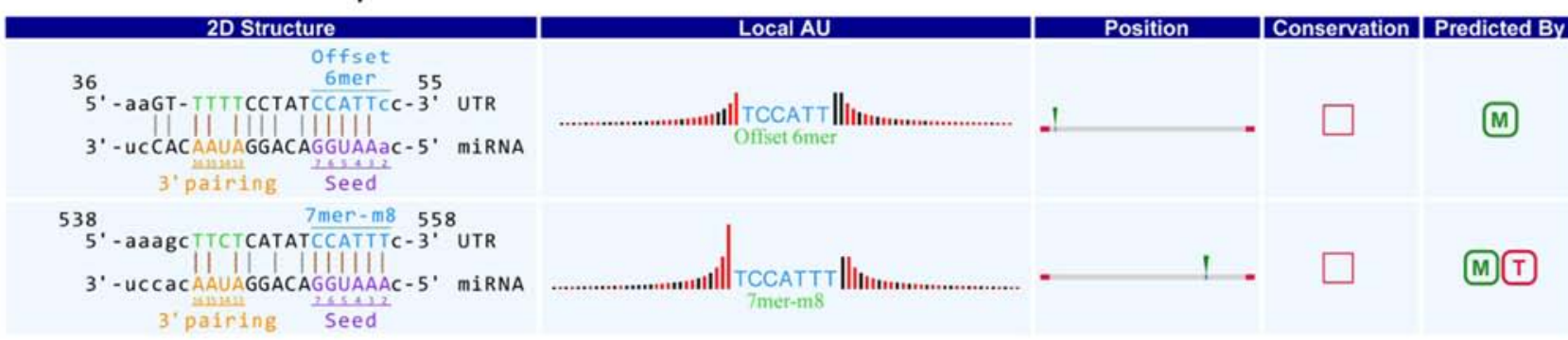

Figure 6. Snippet of the detailed annotation for circRNA/miRNA interactions. Interactions of hsa_circ_0079787/hsa-miR-580-3p, hsa_ circ_0079787/hsa-miR-125b-5p, hsa_circ_0079787/hsa-miR-5191, hsa_circ_0079787/hsa-miR-6830-5p and hsa_circ_0079787/hsa-miR-4694-3p are displayed. UTR, untranslated region; $\mathrm{miRNA}_{\mathrm{miR}}$, microRNA; circRNA/circ, circular RNA; hsa, Homo sapiens; M, circRNA/miRNA interaction target from Arraystar's home-made miRNA target prediction software based on miRanda; T, circRNA/miRNA interaction target from Arraystar's home-made miRNA target prediction software based on TargetScan. 
sensitivity and moderate specificity. In addition, the diagnostic value of the combination of hsa_circ_0079787-HLA-B27 regarding AxSpA vs. HC was superior to that of HLA-B27. Furthermore, the peripheral blood level of hsa_circ_0079787 in patients with $\mathrm{AxSpA}$ was significantly lower than that in patients with SLE and in the ROC analysis, the AUC for distinguishing patients with AxSpA from $\mathrm{HC}+$ SLE subjects was 0.658 with high sensitivity and moderate specificity. These results indicated the potential of hsa_circ_0079787 as a non-invasive diagnostic biomarker for AxSpA, but the diagnostic specificity was not ideal. Sezgin et al (34) demonstrated the diagnostic value of MPV for AS activation. Furthermore, in the present study, it was suggested that the PLT, MPV and PCT in patients with AxSpA were different from those in the controls (SLE + HC) and in the ROC curve analysis, the hsa_circ_0079787-PLT-MPV-PCT combination model had an AUC of 0.838 for distinguishing patients with AxSpA from controls (SLE + HC) with high sensitivity (73.91\%) and specificity $(81.54 \%)$, indicating its high potential as a non-invasive diagnostic biomarker for AxSpA and circRNAs + traditional biomarkers were able to improve the diagnostic value; however, further investigation is still required.

It is well known that circRNAs function as miRNA sponges and regulate target genes, which may have a role in the development of diseases. A bioinformatics analysis identified hsa-miR-580-3p, hsa-miR-125b-5p, hsa-miR-5191, hsa-miR-6830-5p and hsa-miR-4694-3p as potential common targets of hsa_circ_0079787. In previous studies, hsa-miR-125b-5p was reported to correlate with MAPKs and $\mathrm{NF}-\kappa \mathrm{B}$ signaling, which have important roles in $\mathrm{AxSpA}(36,37)$. However, the roles of the other above-mentioned putative miRNAs targets in AxSpA have not been previously reported, to the best of our knowledge. Therefore, further research is required to confirm the role of those other putative miRNA targets in AxSpA and to determine whether hsa_circ_0079787 may have a role in AxSpA by interacting with those putative miRNA targets.

In conclusion, the present study first detected the expression of hsa_circ_0079787 in the blood of patients with AxSpA and SLE, as well as in HC, and indicated that hsa_circ_0079787 was decreased in patients with AxSpA. In addition, hsa_circ_0079787 and the combination of hsa_circ_0079787-PLT-MPV-PCT may serve as novel, non-invasive diagnostic biomarkers for $\mathrm{AxSpA}$, as well as indicators of disease severity and activity. The precise molecular mechanisms underlying the functions of hsa_circ_0079787 in AxSpA still require further investigation.

\section{Acknowledgements}

The authors would like to acknowledge the help in disease diagnosis and case screening of Dr Rui Wu at the Department of Rheumatology, The First Affiliated Hospital of Nanchang University (Nanchang, China).

\section{Funding}

This work was supported by the Key Research and Development Plan Project of Jiangxi Province (grant no. 20181BBG70013), the Science and Technology Plan Project of the Education Department of Jiangxi Province (grant no. GJJ170008), the National Natural Science Foundation of China (grant nos. 81360459 and 81660277), Jiangxi Provincial Natural Science Foundation of China (grant nos. 20151BAB215031 and 20171BAB205113), the Science and Technology Project of Health and Family Planning Commission of Jiangxi Province of China (grant no. 20165094) and the Foundation for Distinguished Young Scientists of Jiangxi Province of China (grant no. 20171BCB23087).

\section{Availability of data and materials}

The data used and/or analyzed during the study are available from the corresponding author on request.

\section{Authors' contributions}

QL, BQF and LZ performed the experiments. YG, ZKH and JML analyzed and interpreted the data. QL and JML made substantial contributions to the design and supervision of the present study and wrote the manuscript. All authors read and approved the final manuscript.

\section{Ethics approval and consent to participate}

This study was authorized by the Ethics Committee of the First Affiliated Hospital of Nanchang University (Nanchang, China). All participants provided written informed consent prior to the initiation of the study.

\section{Patient consent for publication}

Not applicable.

\section{Competing interests}

The authors declare that they have no competing interests.

\section{References}

1. Braun J and Sieper J: Ankylosing spondylitis. Lancet 369: 1379-1390, 2007.

2. Kwan YH, Fong W, How P, Wee HL, Leung YY, Phang JK, Lui NL, Tan CS, Malhotra R, Østbye T and Thumboo J: The impact of axial spondyloarthritis onquality of life (QoL): A comparison with the impact of moderate to end-stagechronic kidney disease on QoL. Qual Life Res 27: 2321-2327, 2018.

3. Prajzlerová K, Grobelná K, Pavelka K, Šenolt L and Filková M: An update on biomarkers in axial spondyloarthritis. Autoimmun Rev 15: 501-509, 2016.

4. Chen LL and Yang L: Regulation of circRNA biogenesis. RNA Biol 12: 381-388, 2015.

5. Hentze MW and Preiss T: circular RNAs: Splicing's enigma variations. EMBO J 32: 923-925, 2013.

6. Salzman J, Chen RE, Olsen MN, Wang PL and Brown PO: Cell-type specific features of circular RNA expression. PLoS Genet 9: e1003777, 2013.

7. Szabo L, Morey R, Palpant NJ, Wang PL, Afari N, Jiang C, Parast MM, Murry CE, Laurent LC and Salzman J: Statistically based splicing detection reveals neural enrichment and tissue-specific induction of circular RNA during human fetal development. Genome Biol 16: 126, 2015.

8. Suzuki H and Tsukahara T: A view of pre-mRNA splicing from RNase R resistant RNAs. Int J Mol Sci 15: 9331-9342, 2014.

9. Hansen TB, Jensen TI, Clausen BH, Bramsen JB, Finsen B, Damgaard CK and Kjems J: Natural RNA circles function as efficient microRNA sponges. Nature 495: 384-388, 2013. 
10. Kumar L, Shamsuzzama, Haque R, Baghel T and Nazir A: circular RNAs: The emerging class of non-coding RNAs and their potential role in human neurodegenerative diseases. Mol Neurobiol 54: 7224-7234, 2017.

11. Wang L, Shen C, Wang Y, Zou T, Zhu H, Lu X, Li L, Yang B, Chen J, Chen S, et al: Identification of circular RNA Hsa circ_0001879 and Hsa_circ_0004104 as novel biomarkers for coronary artery disease. Atherosclerosis 286: 88-96, 2019.

12. Qu S, Liu Z, Yang X, Zhou J, Yu H, Zhang R and Li H: The emerging functions and roles of circular RNAs in cancer. Cancer Lett 414: 301-309, 2018

13. Ouyang Q, Wu J, Jiang Z, Zhao J, Wang R, Lou A, Zhu D, Shi GP and Yang M: Microarray expression profile of circular RNAs in peripheral blood mononuclear cells from rheumatoid arthritis patients. Cell Physiol Biochem 42: 651-659, 2017.

14. Wang F, Nazarali AJ and Ji S: Circular RNAs as potential biomarkers for cancer diagnosis and therapy. Am J Cancer Res 6: 1167-1176, 2016.

15. Lu D and Xu AD: Mini review: Circular RNAs as potential clinical biomarkers for disorders in the central nervous system. Front Genet 7: 53, 2016.

16. Luo Q, Li X, Fu B, Zhang L, Fang L, Qing C, Guo Y, Huang Z and $\mathrm{Li} \mathrm{J}$ : Expression profile and diagnostic value of circRNAs in peripheral blood from patients with systemic lupus erythematosus. Mol Med Rep (In press).

17. Proft F and Poddubnyy D: Ankylosing spondylitis and axial spondyloarthritis: Recent insights and impact of new classification criteria. Ther Adv Musculoskelet Dis 10: 129-139, 2018.

18. Petri M, Orbai AM, Alarcón GS, Gordon C, Merrill JT, Fortin PR, Bruce IN, Isenberg D, Wallace DJ, Nived O, et al: Derivation and validation of the systemic lupus international collaborating clinics classification criteria for systemic lupus erythematosus Arthritis Rheum 64: 2677-2686, 2012.

19. Luo Q, Liu J, Fu B, Zhang L, Guo Y, Huang Z and Li J: Circular RNAs hsa_circ 0002715 and hsa_circ_0035197 in peripheral blood are novel potential biomarkers for new-onset rheumatoid arthritis. Dis Markers 2019: 2073139, 2019.

20. Livak KJ and Schmittgen TD: Analysis of relative gene expression data using real-time quantitative PCR and the 2(-Delta Delta C(T)) methods. Methods 25: 402-408, 2001.

21. Machado P, Landewé R, Lie E, Kvien TK, Braun J, Baker D, van der Heijde D; Assessment of SpondyloArthritis international Society: Ankylosing spondylitis disease activity score (ASDAS): Defining cut-off values for disease activity states and improvement scores. Ann Rheum Dis 70: 47-53, 2011.

22. Garrett S, Jenkinson T, Kennedy LG, Whitelock H, Gaisford P and Calin A: A new approach to defining disease status in ankylosing spondylitis: The bath ankylosing spondylitis disease activity index. J Rheumatol 21: 2286-2291, 1994

23. Huang Z, Su R, Qing C, Peng Y, Luo Q and Li JM: Plasma circular RNAs hsa_circ_0001953 and hsa_circ_0009024 as diagnostic biomarkers for active tuberculosis. Front Microbiol 9: $2010,2018$.

24. Huang Z, Su R, Deng Z, Xu J, Peng Y, Luo Q and Li J: Identifcation of diferentially expressed circular RNAs in human monocyte derived macrophages response to mycobacterium tuberculosis infection. Sci Rep 7: 13673, 2017.
25. Enright AJ, John B, Gaul U, Tuschl T, Sander C and Marks DS MicroRNA targets in Drosophila. Genome Biol 5: R1, 2003.

26. Pasquinelli AE: MicroRNAs and their targets: Recognition, regulation and an emerging reciprocal relationship. Nat Rev Genet 13: 271-282, 2012.

27. Zhang $\mathrm{C}$, Wang $\mathrm{X}$, Chen $\mathrm{Y}, \mathrm{Wu} \mathrm{Z}$, Zhang $\mathrm{C}$ and Shi $\mathrm{W}$ : The down-regulation of hsa_circ_0012919, the sponge for miR-125a-3p, contributes to DNA methylation of CD11a and $\mathrm{CD} 70$ in $\mathrm{CD}^{+} \mathrm{T}$ cells of systemic lupus erythematous. Clin Sci (Lond) 132: 2285-2298, 2018.

28. Li LJ, Zhu ZW, Zhao W, Tao SS, Li BZ, Xu SZ, Wang JB, Zhang MY, Wu J, Leng RX, et al: Circular RNA expression profile and potential function of hsa_circ_0045272 in systemic lupus erythematosus. Immunology 155: 137-149, 2018.

29. Luo Q, Zhang L, Li X, Fu B, Deng Z, Qing C, Su R, Xu J, Guo Y, Huang Z and Li J: Identification of circular RNAs hsa_circ_0044235 in peripheral blood as novel biomarkers for rheumatoid arthritis. Clin Exp Immunol 194: 118-124, 2018.

30. Miao Q, Zhong Z, Jiang Z, Lin Y, Ni B, Yang W and Tang J: RNA-seq of circular RNAs identified circPTPN22 as a potential new activity indicator in systemic lupus erythematosus. Lupus 28: 520-528, 2019.

31. Ouyang Q, Huang Q, Jiang Z, Zhao J, Shi GP and Yang M: Using plasma circRNA_002453 as a novel biomarker in the diagnosis of lupus nephritis. Mol Immunol 101: 531-538, 2018.

32. Chen Q, Chen DY, Xu XZ, Liu YY, Yin TT and Li D: Platelet/lymphocyte, lymphocyte/monocyte, and neutrophil/lymphocyte ratios as biomarkers in patients with rheumatoid arthritis and rheumatoid arthritis-associated interstitial lung disease. Med Sci Monit 25: 6474-6481, 2019.

33. Wang F, Yan CG, Xiang HY, Xing T and Wang NS: The significance of platelet activation in ankylosing spondylitis. Clin Rheumatol 27: 767-769, 2008

34. Sezgin M, Tecer D, Kanık A, Kekik FS, Yeşildal E, Akaslan E, Yildırım G and Sahin G: Serum RDW and MPV in ankylosing spondylitis: Can they show the disease activity? Clin Hemorheol Microcirc 65: 1-10, 2017.

35. Liu J, Cao Y, Huang C, Wang Y, Chen X, Zhang W, Wang G, Fan H, Ge Y, Chen R, et al: Use of xinfeng capsule to treat abarticular pathologic changes in patients with rheumatoid arthritis. J Tradit Chin Med 34: 532-538, 2014.

36. Rasheed Z, Rasheed N, Abdulmonem WA and Khan MI: MicroRNA-125b-5p regulates IL-1 $\beta$ induced inflammatory genes via targeting TRAF6-mediated MAPKs and NF- $\mathrm{BB}$ signaling in human osteoarthritic chondrocytes. Sci Rep 9: 6882, 2019.

37. Liu Y, Ye Z, Li X, Anderson JL, Khan M, DaSilva D, Baron M, Wilson D, Bocoun V, Ivacic LC, et al: Genetic and functional associations with decreased anti-inflammatory tumor necrosis factor alpha induced protein 3 in macrophages from subjects with axial spondyloarthritis. Front Immunol 8: 860, 2017.

This work is licensed under a Creative Commons Attribution-NonCommercial-NoDerivatives 4.0 International (CC BY-NC-ND 4.0) License. 